\title{
Experimental Investigation of the Optical Injection Locking Dynamics in Single-Section Quantum-Dash Fabry-Pérot Laser Diode for Packet- Based Clock Recovery Applications
}

\author{
Ramón Maldonado-Basilio, Josué Parra-Cetina, Sylwester Latkowski, Nicola \\ Calabretta, and Pascal Landais,
}

R. Maldonado-Basilio was with The RINCE Institute, School of Electronic Engineering, Dublin City University, Glasnevin, Dublin 9, Dublin, Ireland. He is now with the Center for Research in Photonics, University of Ottawa, Ottawa, ON Canada K1N6N5 (e-mail: rmaldon2@uottawa.ca).

Parra-Cetina, and P. Landais are with The RINCE Institute, School of Electronic Engineering, Dublin City University, Glasnevin, Dublin 9, Dublin, Ire-land (e-mail: josue.parracetina2@mail.dcu.ie; landaisp@eeng.dcu.ie).

S. Latkowski and N. Calabretta are with the Eindhoven University of Technology, Department of Electrical Engineering, 5600MB Eindhoven, The Netherlands (e-mail: s.latkowski@tue.nl; n.calabretta@tue.nl).

This work was supported by Enterprise Ire-land, EU/ERDF, under Research Project TD/2009/0303 and the Higher Education Authority via the INSPIRE program.

\begin{abstract}
An experimental study of the dynamics of a quantum-dash Fabry-Pérot passively mode-locked laser diode is presented. Firstly, the switching on and off characteristic times of the mode-locking mechanism with pulsed biasing current are assessed. Secondly, the locking and unlocking characteristic times to the injection of a 10 -Gbps pseudo-random binary sequence of nonreturn-to-zero data are determined. The dynamics is analysed through the instantaneous frequency of the $\sim 40 \mathrm{GHz}$ beat-tone signal measured at the output of the laser under investigation, which after a frequency down-conversion stage, is recorded by a real-time oscilloscope. Experimental results indicate that a time of ns characterizes the establishment of the passive mode-locking mechanism for a pulsed biasing current. A time of 20 ns has been measured for the synchronization of the quantum-dash laser diode to the injected 10 Gbps data sequence. In addition, the mode-locked laser diode de-synchronizes and switches to the free-running condition in also 20 ns after a holding time of $\mathbf{1 0 0}$ ns.
\end{abstract}

Index Terms-All-optical clock recovery, mode-locked semiconductor lasers, packet-based clock recovery, quantum-dot semiconductor lasers. 


\section{INTRODUCTION}

The exponential growth of data traffic in access networks makes it likely that future optical metro and core telecommunication systems should be capable of handling tens of Tbps data rates. To cope with it, links interconnecting the nodes of the network shall carry optical packets at transmission rates above $100 \mathrm{Gbps}$. Assuming packets typically comprised of 1500 bytes and transmitted at such data rate results in time slots of $120 \mathrm{~ns}$ per packet. To optimize the load at which such a system can operate, the guard times between the packets have to be small, in the order of a few tens of nanoseconds. A system with an asynchronous packet based clock extraction circuitry at the receiver end operating at very fast locking and unlocking times (few tens of $n s$ ) is therefore an essential building block required to implement several functionalities for high speed optical processing such as ultrafast optical time demultiplexers, high speed burst mode receivers, optical header processors, and $3 \mathrm{R}$ regenerators.

All-optical packet-based clock recovery (CR) has therefore been an extensive topic of research. Among a variety of different techniques, CR circuits based on electronic -locked loops and mode-locked ring-lasers exhibit a relatively long time for synchronization and are not suitable for asynchronous short optical packets. Self-pulsating lasers (SPL) require less overhead for clock acquisition in asynchronous packets. For instance, a synchronization time of 1 ns has been reported after extracting an inline bit rate clock signal from $10 \mathrm{Gbps}$ data packets using a SPL based on a distributed Bragg reflector with detuned gratings [1]. Packet based clock recovery approaches featuring a faster response have also been investigated utilizing low finesse Fabry-Pérot filters (FPF). An instantaneous synchronization and a 400 ps persistence time (or de-synchronization) from short and closely spaced optical packets at $40 \mathrm{Gbps}$ has been obtained using a FPF followed by an ultrafast nonlinear interferometer based on a semiconductor optical amplifier (SOA) [2]. A photonic integrated version of this concept but utilizing a Mach-Zehnder interferometer was reported in [3]. Exploiting cross-gain modulation in an SOA, optical clock at $40 \mathrm{GHz}$ has been recovered from ultrafast $640 \mathrm{Gbps}$ data signals [4]. Unlike the aforementioned experiments with on-off keying (OOK) return-to-zero (RZ) pulsed data streams, inline bit rate optical clock has also been extracted from $10 \mathrm{Gbps}$ nonreturn-to-zero (NRZ) differential phase shift keying data utilizing a FPF followed by an SOA gate [5]. Instantaneous synchronization and desynchronization was obtained. Despite the remarkable achievements in terms of ultrafast synchronization and de-synchronization times, the complexity, bulkiness, and limited operation speed of some experimental demonstrations, in addition to a reduction of the components count (which eventually translates into less energy consumption for operation) seem to be motivating aspects for further improvement.

Owing to their small size, low energy consumption, and low cost, passively mode-locked semiconductor lasers (PMLL) based on quantum dots/dash (QD/QDash) structures have been investigated for all-optical synchronous clock recovery operations. The first demonstration of inline bit rate $\mathrm{CR}$ at $40 \mathrm{GHz}$ using a QDash Fabry-Pérot (FP) laser was reported in [6]. From recent investigations, it has also been shown that a QDash PMLL laser can be externally synchronized to the optical injection of harmonic pseudo-random binary sequences (PRBS) of RZ pulses from 42 to $425 \mathrm{Gbps}$ [7], [8], as well as to the sub-harmonic and harmonic RZ and NRZ data signals from 10,20,40,80 and $160 \mathrm{GHz} / \mathrm{Gbps}$ [9], [10]. The recovered synchronized signal comprises stable $40 \mathrm{GHz}$ clock pulses featuring a pulse-width of $1.8 \mathrm{ps}$ and sub-picosecond timing jitter. As reported in [11], the small timing jitter exhibited by the QDash PMLL diode has benefited the implementation of all-optical clock recovery and re-timing operations by optically filtering the recovered clock and carrying out cross-phase modulation with an RZ-OOK $40-G b p s$ data signal in a highly nonlinear fiber. In addition, a reduction from tens of kilohertz to less than $10 \mathrm{~Hz}$ in the linewidth of the $40-\mathrm{GHz}$ beat-tones is observed at synchronized conditions relative to its free-running operation [10]. The main advantage of implementing alloptical clock recovery based on QDash FP mode-locked lasers relative to SPL approaches relies on the fact that the former devices are able to produce a train of short optical clock pulses both in free-running and in optical external injection conditions. At the receiver end of an optical time division multiplexing system, these sub-picosecond clock pulses can be used for optically demultiplexing high speed data streams at bit rates 
beyond $160 \mathrm{Gbps}$. Indeed, at such speeds, only devices featuring sub-picosecond switching window can be employed as all-optical demultiplexers [4].

The aim of this work is to present detailed experimental investigations of the fast dynamic characteristics of the QDash FP passively mode-locked laser for all-optical packed based clock recovery applications. To the best of the authors knowledge, this dynamic aspect of the CR based on QDash FP laser has not been reported to date. Firstly, the switching on and off characteristic times of the mode-locking mechanism with pulsed biasing current are assessed. Secondly, the locking and unlocking characteristic times to the injection of a pseudorandom binary sequence of NRZ data are determined. It is worth mentioning that the locking and unlocking times have been typically measured through the use of optical sampling oscilloscopes: a clear trace on the oscilloscope is displayed only for synchronized signals, whilst non-synchronized pulses lead to a nearly random distribution of the measuring points. The synchronization is hence detected by the transition from an undefined distribution of points to a clear and sharp pulse trace. Contrary, the approach proposed in this work is based on the concept presented in [12] and focuses on an analysis of the instantaneous frequency of the $\sim 40 \mathrm{GHz}$ beat-tone signal measured at the output of the laser under investigation, which after a frequency down-conversion (FDC) stage, is recorded by a real-time oscilloscope (RTO).

Regarding the dynamics with pulsed biasing current, the appearance of a beat-tone with a quasi-constant center frequency determines the switching on time of the QDash PMLL diode whilst it operates in freerunning conditions. Furthermore, once the laser diode is biased with a constant current, a transition of the beat-tone frequency that is measured at free- running conditions to the frequency at synchronized conditions determines the locking time associated to the optical external synchronization.

\section{EXPERIMENTS AND RESULTS}

The device under investigation is a $1 \mathrm{~mm}$ long, unpack-aged single-section QDash Fabry-Pérot laser diode, with no saturable absorber section. A detailed description of the QDash-based structure, as well as the dimensions of the active core can be found in [13], [14]. This passively mode -locked laser diode presents a bias threshold of $18 \mathrm{~mA}$ at $25 \circ \mathrm{C}$, and provides an average optical power of $0.4 \mathrm{~mW}$ at $100 \mathrm{~mA}$ when coupled to a lensed single mode fiber. It features more than 40 longitudinal modes with $0.31 \mathrm{~nm}$ intermodal separation, resulting in a $3 \mathrm{~dB}$ optical bandwidth of $12 \mathrm{~nm}$ centered at $1526 \mathrm{~nm}$ [9], [10]. In free-running conditions, the QDash PMLL exhibits optical pulses featuring a width of $720 \mathrm{ps}$ and a timing jitter of $150 \mathrm{~s}$ after a passive compression scheme [15]. A further detailed investigation of the dispersion compensation in this type of lasers to achieve the generation of picosecond pulses has been recently discussed in [16], [18].

\section{A. Locking Dynamics With Pulsed Biasing Current}

The experimental setup to assess the switching on and off characteristic times when the biasing current of the QDash FP-PMLL diode is turning on and off is depicted in Fig. 1. Pulsed bias current is provided by a synthesized clock generator set at $40 \mathrm{kHz}$ and $50 \%$ duty cycle, supplying low and high current levels of 0 and $100 \mathrm{~mA}$, respectively. The selected frequency of the clock generator allows for an efficient use of the RTO in terms of its internal buffer size and sampling rate, whereas it is sufficient to observe the dynamic behaviour of the tested device. Moreover, the selection of the bias current high level relies on the operating conditions of the QDash PMLL at which it synchronizes to an externally injected signal [9]. The optical power emitted from the laser is collected through a lensed fiber and the $\sim 40 \mathrm{GHz}$ beat-tone signal is detected on a fast $(50 \mathrm{GHz})$ photodetector (PD) followed by an RF-amplifier. Taking into account the features of the RTO used in this experiment ( $10 \mathrm{GHz}$ frequency bandwidth and $40 \mathrm{GSa} / \mathrm{s}$ sampling rate), the beat-tone signal of interest is frequency down-converted to $1 \mathrm{GHz}$ by using an RF-mixer and a local oscillator (LO) at $39 \mathrm{GHz}$. The signal from 
the LO features a linewidth of a few hertz, affecting to a minimal extent the characteristics of the $40 \mathrm{GHz}$ beat-tone after the RF-mixer. A trace of the $1 \mathrm{GHz}$ FDC beat-tone signal measured at the mixer output with an electrical spectrum analyzer (ESA) is depicted in the inset of Fig. 1 whilst the PMLL diode is DC-biased at 100 $\mathrm{mA}$. Free-running operation (no external optical injection) of the QDash PMLL diode is analyzed whilst it is DCbiased at $100 \mathrm{~mA}$. Fig. 2(a) shows the FDC beat-tone measured with the RTO. A stable frequency component of $\sim 1 \mathrm{GHz}$ is clearly observed in this signal, as depicted in the zoom-in trace of Fig. 2(b) and the spectrum of Fig. 2(c). Then the QDash PMLL is operated with a $40 \mathrm{kHz}$ pulsed bias current as shown in Fig. 2(d). From the zoom-in plots depicted in Fig. 2(e) and (f), a rising time (from 10\% to 90\%) and falling time (from 90\% to $10 \%$ ) of 41 and 39 ns are measured on the biasing current function, respectively. The time domain trace of the FDC beat-tone is shown in Fig. 2(g). Analyzing the power emitted from the QDash PMLL diode, visualized in these plots as the envelope of the beat-tone, it does not exhibit an instantaneous response with the current supplied. Relative to the time when the threshold current $\left(I_{\mathrm{th}}\right)$ is supplied, a switch on time $\tau_{\text {of }}$ around 30 ns is observed, as revealed in the zoom-in trace shown in Fig. 2(h). In order to determine the passive modelocking characteristic time relative to the point where the $90 \%$ of the supplied bias current is reached (which occurs at $41 \mathrm{~ns}$ ), the instantaneous frequency exhibited by the recorded FDC beat-tone is analyzed. In this case, a fast-Fourier Transform (FFT) is implemented to the recorded data in time windows of 10 ns. Starting from the initial recorded time, the analyzed time window is shifted in steps of 2 ns until the data in the full time span is computed. As depicted in Fig. 2(j) and the zoom-in of Fig. 2(k), the results of this analysis show the frequency of the down converted beat-tones stabilizes to a constant value of $\sim 1 \mathrm{GHz}$ only after a response time of $\sim 46 \mathrm{~ns}$. It indicates that the passive mode-locking process taking place in the QDash PMLL occurs $\sim \overline{\mathrm{ns}}$ after the $90 \%$ of the pulsed biasing current is reached. Following the same approach, frequency unstable beat-tones are retrieved and shown in Fig. 2(I) after the supplied current is lower than 90 mA. It means the passive mode-locking process exhibits an immediate response while switching off the bias current, only determined by its decreasing slope. It is worth mentioning that to obtain the passive mode-locking characteristic times with the supplied pulsed current reported in this work, it has been taken into account the time delay experienced by the electric signals biasing the QDash PMLL (ie, from the clock synthesizer to the laser $\mathrm{p}-\mathrm{n}$ junction) and the synchronized beat-tone (ie, from the QDash PMLL output to the real-time oscilloscope).

\section{B. Locking Dynamics With Injection of 10-Gbps PRBS NRZ Data Sequences}

Here, the locking dynamics of the QDash laser with the injection of 10 Gbps nonreturn-to-zero pseudorandom binary sequences whilst it is DC-biased at $110 \mathrm{~mA}$ are investigated. Experimental setup is depicted in Fig. 3. A tunable external cavity laser (ECL) set at $1540 \mathrm{~nm}$ is intensity modulated by 10-Gbps PRBS NRZ data signals and injected to the QDash PMLL diode after amplification and filtering stages. The generation of the $50-\mu_{\mathrm{S}}$-long packets is implemented by a built-in feature of the $\mathrm{ECL}$, applying a direct modulation at $20 \mathrm{kHz}$ through an embedded electrical square pulse signal. At the upper level of the modulating signal, an average power of $+6 j \mathrm{dBm}$ (measured at VOA -1) is injected to the QDash laser diode, which is high enough to allow an external synchronization to the PRBS NRZ data signal. At the lower level of the modulating signal, only around $12 \mathrm{dBm}$ (measured at VOA -1 and supplied from the amplified spontaneous emission of the EDFA) are injected to the QDash laser. In this case, synchronization of the mode-locked laser diode to the injected signal is not achieved, and hence it operates in free-running conditions. The optical data signal is amplified and filtered by a 2-nm optical band-pass filter (OBPF-1) centered at the wavelength of the ECL. After injection to the QDash laser diode, the recovered clock signal is amplified and filtered by OBPF- 2, which features a bandpass of $5 \mathrm{~nm}$ and center wavelength of $1530 \mathrm{~nm}$. Synchronized (or de-synchronized) clock pulses are analyzed via an electrical spectrum analyzer, a real-time oscilloscope, and a sampling oscilloscope after photodetection stage. Injected PRBS NRZ data and recovered clock pulses are also recorded at the input and output of the QDash laser by an optical sampling oscilloscope, respectively. In this case, the output clock from the PRBS generator is connected to the trigger signal of the sampling oscilloscope.

As shown in Fig. 4, special care has been taken for optimizing the signal injection to the QDash laser diode. The spectrum for free-running is depicted in the red traces of Fig. 4(a) and the zoom-in of Fig. 4(b). In this 
condition, the injected signal is negligible and only the profile of OBPF -1 is observed after the amplified spontaneous emission of the EDFA partially passes through. The spectrum for synchronization is illustrated in the blue traces of Fig. 4(a) and the zoom-in of Fig. 4(b). A gain compression in the emission of the QDash laser diode resulting from the external injection is observed. To achieve synchronization, the center wavelength of the injected signal must match at a large extent one of the Fabry-Pérot weak resonances far away from the QDash laser diode emission. In addition, four-wave mixing (FWM) side bands in coincidence with adjacent FP resonances ( $40 \mathrm{GHz}$ apart) appear in the optical spectrum when external synchronization is achieved. The experimental observations aforementioned are justified by the mechanism behind the mode- locking in a FP semiconductor laser. In free-running operation, the passive mode-locking results from intra-cavity FWM processes occurring between the adjacent longitudinal modes across the gain spectrum of the laser. These processes are responsible of the phase correlation between the optical modes

Owing to the fact that the free-spectral range of the device under investigation (which is primary determined by the cavity length of the laser) is equal to $40 \mathrm{GHz}$, the dominant FWM process is governed by carrier density pulsation. In op-tical external synchronization conditions, the free- running beat tone generated by the laser is locked to the bit-rate fundamental and harmonic frequencies of the optical incoming data signal, providing the injected frequencies lie within the locking range of the laser.

Dynamics of the QDash mode -locked laser diode is experimentally analyzed by switching on and off the injected data stream at a frequency of $20 \mathrm{kHz}$. Note that the bit rate of the injected NRZ data stream is set at $10 \mathrm{Gbps}$, whilst the free-running frequency of the beat-tone occurs at $40 \mathrm{GHz}$. It im-plies the QDash laser is synchronized to the third harmonic of the incoming signal spectrum, which is not as efficient as a $40 \mathrm{Gbps}$ data stream. A period of the modulating signal is illustrated in Fig. 5(a) after being recorded by the RTO. A zoom-in of this trace is depicted in Fig. $5(\mathrm{~b})$ and (c), where a rising (from $10 \%$ to $90 \%$ ) and falling time (from $90 \%$ to $10 \%$ ) of 100 and 25 ns are retrieved, respectively. As shown in Fig. 5(d), a change in the amplitude of the recorded FDC beat tones is ob-served whilst switching from free-running to external synchronization and vice-versa. Unlike the free-running operation, the FDC beat tones exhibit an amplitude reduction while the QDash mode- locked laser is externally synchronized. In addition, relative to the 100 -ns mark imposed by the rising time of the electrical modulating signal applied to the ECL, synchronization of the QDash laser to the injected data signal is achieved after a switch on time of $20 \mathrm{~ns}$, as depicted in Fig. 5(e). Once the synchronization signal is switched off, the mode- locked laser diode switches to the free-running condition in $20 \mathrm{~ns}$ after a holding time of $\sim 100 \mathrm{~ns}$ (Fig. 5(f)). Note that the $100 \mathrm{~ns}$ turn on time of the directly modulated external cavity laser obeys to the response of its embedded modulation functionality illustrated in Fig. 5(b). The ECL emission turns on only after the $90 \%$ of the relative amplitude of the internal control signal is reached. Until the 100-ns mark, the input optical power at the QDash PMLL diode is very small (only the filtered amplified spontaneous emission of the EDFA). For this reason, in Fig. 5(e) it is not observed any transition from free- running to synchronization of the QDash laser until after the $100 \mathrm{~ns}$ mark is reached. This also indicates that there is no holding time for this transition. As illustrated in Fig. 5(e), a clear transition from free-running to external synchronization is exhibited by the beat-tone signal relative to this mark reference, making possible the assessment of the synchronization time. The reported dynamics in the QDash PMLL diode for packet based all-optical clock recovery are hence conclusive.

At $10 \mathrm{Gbps}$, the switching on and off characteristic times indicate that the QDash laser diode synchronizes after 200 bits of the incoming data signals. Furthermore, a holding time of $100 \mathrm{~ns}$ implies the device under investigation can remain in a synchronized condition after a train of 1000 consecutive zero-data bits. Reported synchronization and de-synchronization times are retrieved after taking into account the delay from the electric modulating signal to the RTO relative to the delay from the output of the ECL to the input of the photodiode plus the delay from this point to the RTO.

Illustrated in Fig. 6 are the two frequency components retrieved after computing the FFT from the $50-\mu_{\mathrm{s}}$-long recorded signal of the FDC beat-tones: a spectral component is retrieved with the normalized frequency down converted beat-tones illustrated in Fig. 5, the spectral analysis reveals a reduction of the amplitude in 
synchronization (component around $1 \mathrm{GHz}$ ) relative to the free-running (component around $1.01 \mathrm{GHz}$ ). In addition, the beat-tone retrieved for synchronization features a larger linewidth, which in this case is a result of a frequency drift (in the order of a few megahertz) of the external cavity laser utilized in this experiment whilst operating in direct modulation mode.

\section{CONCLUSION}

Dynamic characteristics of a single-section QDash PMLL diode have been investigated. A retrieved time of $5 \mathrm{~ns}$ characterizes the establishment of the passive mode-locking mechanism for a pulsed biasing current from 0 to $100 \mathrm{~mA}$. In addition, a retrieved time of $20 \mathrm{~ns}$ has been measured for the synchronization

of the QDash mode-locked laser diode to an injected 10- Gbps PRBS NRZ data sequence. Furthermore, the mode-locked laser diode de-synchronizes and switches to the free-running condition in also $20 \mathrm{~ns}$ after a holding time of $\sim 100 \mathrm{~ns}$. Retrieved results provide a consistent study of the QDash mode-locked laser diode dynamics and encourage the implementation of further experiments with data packets featuring a higher transmission rates, as well as different length and separation between packets. Moreover, the fact that a single device (the QDash PMLL diode) is used for packet-based clock recovery suggests the components count and hence the energy consumption of this functionality within an all-optical communication system can be substantially minimized. 


\section{REFERENCES}

S. Bauer, C. Bornholdt, O. Brox, D. Hoffmann, M. Mohrle, G. Sahin, Sartorious, S. Schelhase, B. Lavigne, and D. Chiaroni, "Ultrafast locking optical clock for IP packet switching applications," in Proc. Opt. Fiber Conf., 2000, paper TuF5-1.

E. Kehayas, L. Stampoulidis, H. Avramopoulos, Y. Liu, E. Tangdiongga, and H. J. S. Dorren, " $40 \mathrm{~Gb} / \mathrm{s}$ all-optical packed clock recovery with ultrafast lock-in time and low inter-packet guardbands," Opt. Exp., vol. 13, no. 2, pp. 475-480, 2005.

E. Kehayas, D. Tsiokos, P. Bakopoulos, D. Apostolopoulos, D. Pentrantonakis, L. Stampoulidis, A. Poustie, R. McDougall, G. Maxwell,

Liu, S. Zhang, H. J. S. Dorren, J. Seoane, P. Van Holm-Nielsen, Jeppesen, and $\mathrm{H}$. Avramopoulos, " $40-\mathrm{Gb} / \mathrm{s}$ all-optical processing systems using hybrid photonic integration technologies," J. Lightw. Technol., vol. 24, no. 12, pp. 4903-4911, Dec. 2006.

E. Tangdiongga, H. C. Hansen Mulvad, H. de Waardt, G. D. Khoe, M. J. Koonen, and H. J. S. Dorren, "SOA-based clock recovery and demultiplexing in a lab trial of $640-\mathrm{Gb} / \mathrm{s}$ OTDM transmission over 50-km fibre link," in Proc. ECOC, 2007, postdeadline paper PD 1.2.

M. Presi, N. Calabretta, G. Contestabile, and E. Ciaramella, "Wide dynamic range all-optical clock and data recovery from preamble-free NRZ-DPSK packets," IEEE Photon. Technol. Lett., vol. 19, no. 6, pp. 372-374, Mar. 2007.

J. Renaudier, B. Lavigne, M. Jourdran, P. Gallion, F. Lelarge, B. Da-gens, A. Accard, O. Legouezigou, and G.-H. Duan, "First demonstration of all-optical clock recovery at $40 \mathrm{GHz}$ with standard-compliant jitter characteristics based on a quantum-dots self-pulsating semiconductor laser," in Proc. ECOC, 2005, paper Th4.3.4.

V. Roncin, A. O'Hare, S. Lobo, E. Jacquette, L. Bramarie, P. Rochard, Q.-T. Le, M. Gay, J.-C. Simon, A. Shen, J. Renaudier, F. Lelarge, and G.-H. Duan, "Multi-data-rate system performance of a 40-GHz all-op-tical clock recovery based on a quantum-dot Fabry-Pérot laser," IEEE Photon. Technol. Lett., vol. 19, no. 19, pp. 14091411, Oct. 2007.

M. Costa e Silva, A. Lagrost, L. Bramerie, M. Gay, P. Besnard, M. Joindot, J. C. Simon, A. Shen, and G. H. Duan, "Up to $425 \mathrm{GHz}$ all optical frequency down-conversion clock recovery based on quantum dash Fabry-Perot mode-locked laser," in Proc. OFC, 2010, postdead-line paper PDPC4.

R. Maldonado-Basilio, S. Latkowski, S. Philippe, and P. Landais, "Ex-perimental investigation of harmonic and subharmonic synchroniza-tion of $40 \mathrm{GHz}$ mode-locked quantum-dash laser diodes," Opt. Lett., vol. 36, no. 9, pp. 1569-1571, 2011.

R. Maldonado-Basilio, S. Latkowski, S. Philippe, and P. Landais, " $40 \mathrm{GHz}$ mode-beating with $8 \mathrm{~Hz}$ linewidth and 64 fs timing jitter from a synchronized mode-locked quantum-dash laser diode," Opt. Lett., vol. 36, no. 16, pp. 3142-3144, 2011.

X. Tang, S. H. Chung, A. S. Karar, J. C. Cartledge, A. Shen, and G.-H. Duan, "Spectral filtering characteristics of a quantum-dash Fabry-Pérot laser and its implications on $40 \mathrm{~Gb} / \mathrm{s}$ XPM based optical retiming per-formance," J. Lightw. Technol., vol. 29, no. 1, pp. 31-36, Jan. 2011. 
R. Maldonado-Basilio, J. Parra-Cetina, S. Latkowski, P. Landais, and N. Calabretta, "Mode-locking dynamics in a quantum-dash Fabry-Pérot laser diode for packet based clock recovery applications," in Proc. OFC, 2012, paper JTh2A.

F. Lelarge, B. Dagens, J. Renaudier, R. Brenot, A. Accard, F. van Dijk, D. Make, O. Le Gouezigou, J.-G. Provost, F. Poingt, J. Lan-dreau, O. Drisse, E. Derouin, B. Rousseau, F. Pommereau, and G.-H. Duan, "Recent advances on InAs/InP quantum dash based semicon-ductor lasers and optical amplifiers operating at $1.55 \mu \mathrm{m}, "$ IEEE J. Sel. Topics Quantum Electron., vol. 13, no. 1, pp. 111-124, Jan.-Feb. 2007.

G.-H. Duan, A. Shen, A. Akraut, F. Van Dijk, F. Lelarge, F. Pom-mereau, O. LeGouzegou, J.-G. Provost, H. Gariah, F. Blache, F. Mal-lecot, K. Merghem, A. Martinez, and A. Ramdane, "High performance InP-based quantum dash semiconductor mode-locked lasers for optical communications," Bell Labs Tech. J., vol. 14, no. 3, pp. 6384, 2009.

R. Maldonado-Basilio, J. Parra-Cetina, S. Latkowski, and P. Landais, "Timing jitter, optical, and mode-beating linewidths analysis on subpi-cosecond optical pulses generated by a quantum-dash passively mode-locked semiconductor laser," Opt. Lett., vol. 35, no. 8, pp. 1184-1186, 2010.

R. Rosales, S. C. Murdoch, R. T. Watts, K. Merghem, A. Martinez, F. Lelarge, A. Accard, L. P. Barry, and A. Ramdane, "High performance mode locking characteristics of single section quantum dash lasers," Opt. Exp., vol. 20, no. 8, pp. 8649-8657, 2012.

S. Latkowski, R. Maldonado-Basilio, and P. Landais, "Sub-picosecond pulse generation by $40-\mathrm{GHz}$ passively mode-locked quantum-dash 1-mm-long Fabry-Perot laser diode," Opt. Exp., vol. 17, no. 21, pp. 19166-19172, Oct. 2009.

J. Renaudier, G.-H. Duan, P. Landais, and P. Gallion, "Phase corre-lation and linewidth reduction of $40 \mathrm{GHz}$ self-pulsation in distributed Bragg reflector semiconductor lasers," IEEE J. Quantum Electron., vol. 43, no. 2, pp. 147-156, Feb. 2007. 


\section{Figures}

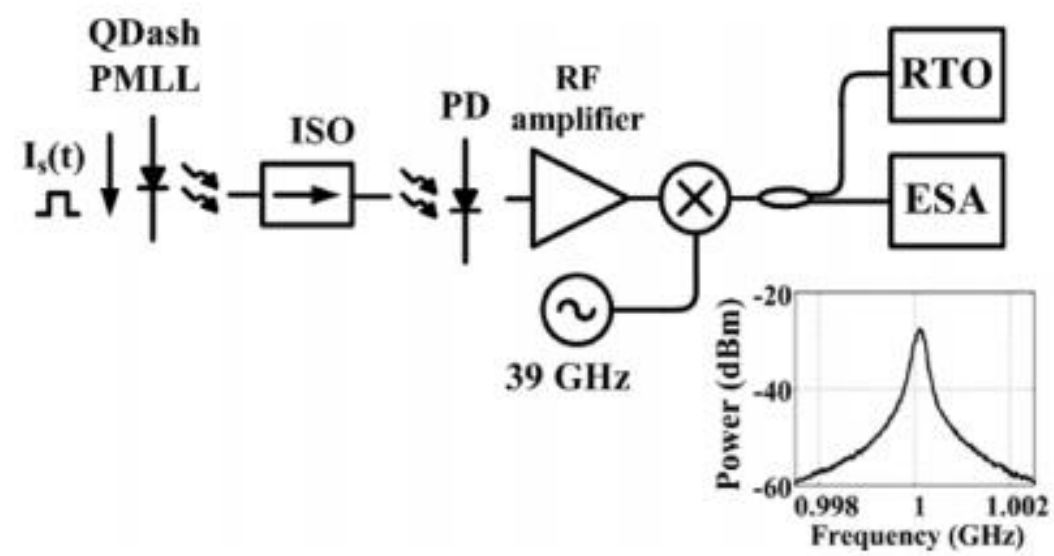

Fig. 1. Experimental setup for assessing the switching time of the passive mode-locking mechanism by turning on and off the biasing current. Inset: frequency down-converted beat-tone measured at the output of the RFmixer. ISO: optical Isolator. PD: photo-detector. RF: radio frequency. RTO: real-time oscilloscope. ESA: electrical spectrum analyzer. 

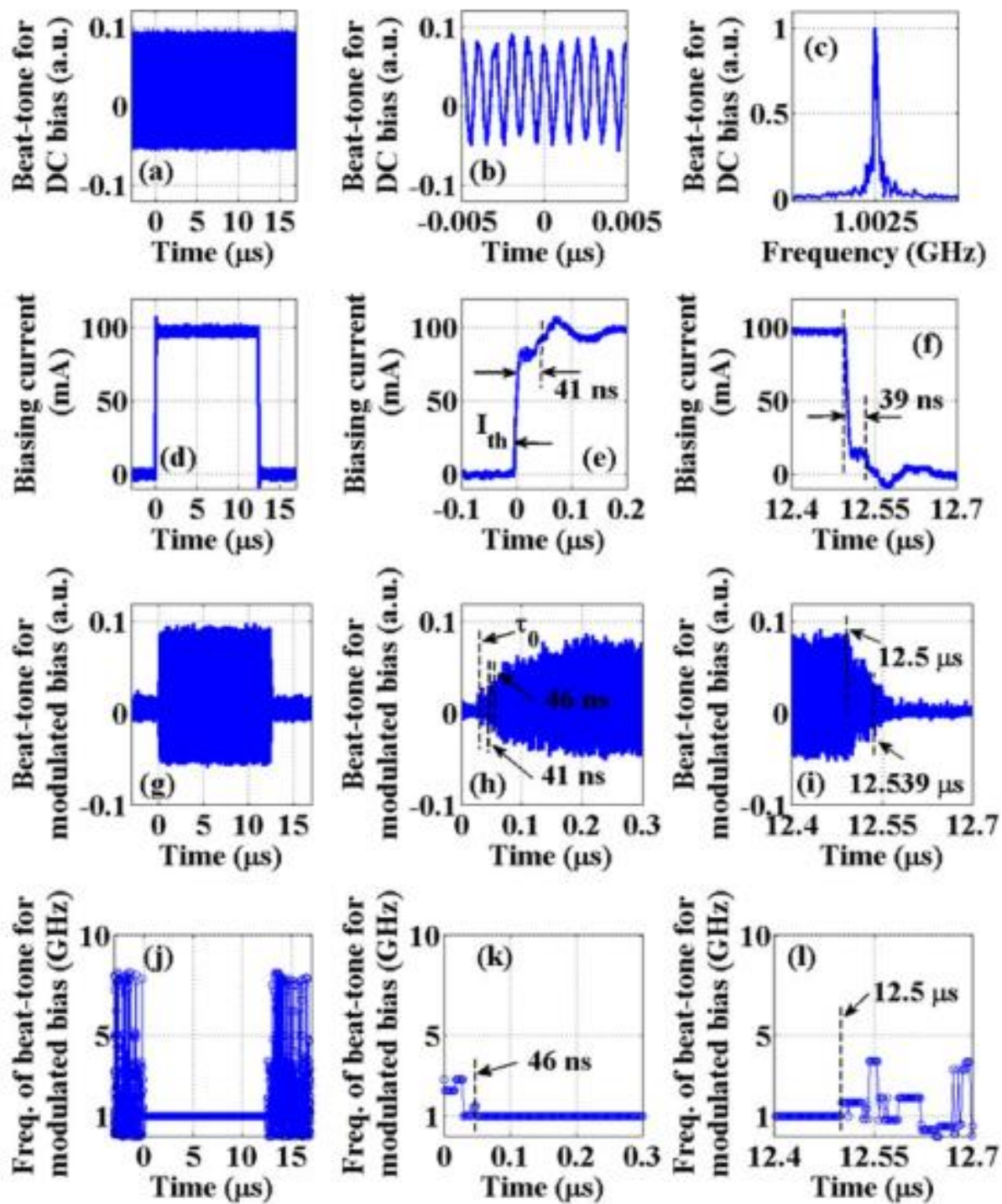

Fig. 2. Frequency down-converted beat-tone in constant current conditions.(a) Full span. (b) Zoom-in trace. (c) FFT trace. Time-domain traces of the modulating bias current source: (d) full span, (e)-(f) rising and falling times, respectively. Frequency down-converted beat-tone after applying the modulating bias current: (g) full span, (h)-(i) zoom-in for passive locking and unlocking assessment, respectively. Instantaneous frequency of the beat-tone: (j) full span and-(I) zoom-in for passive locking and unlocking assessment, respectively. 


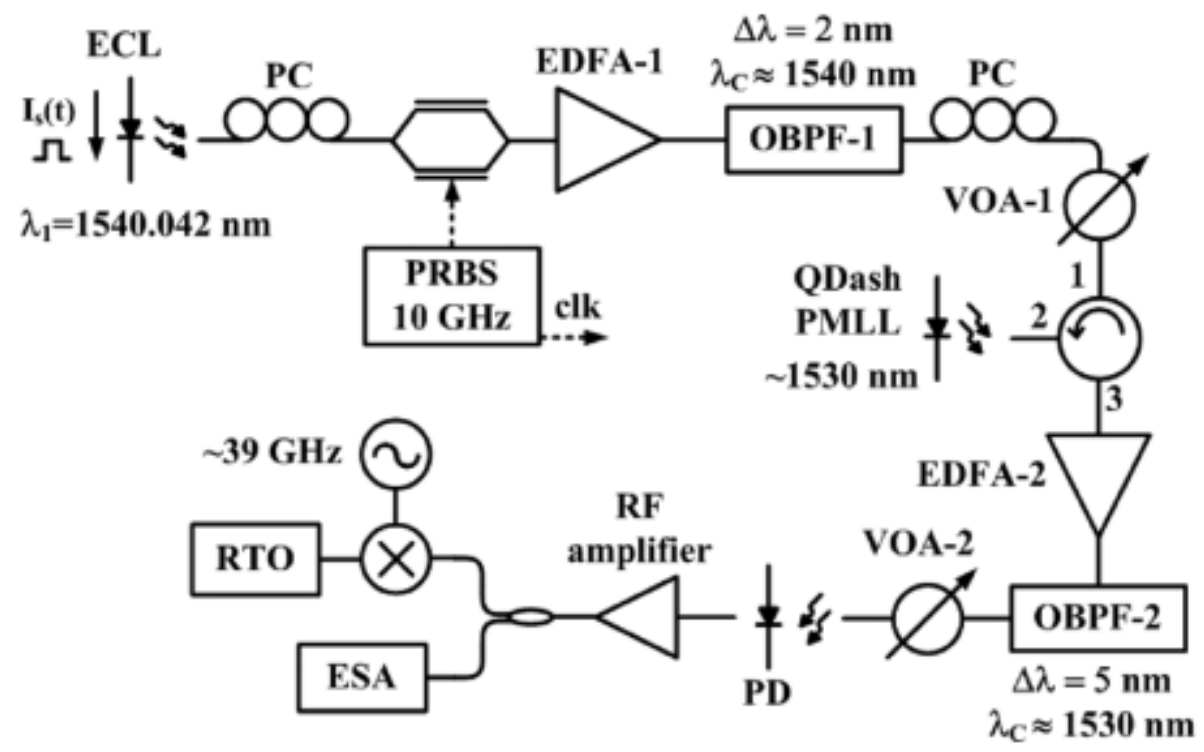

Fig. 3. Experimental setup to assess the dynamics of the QDash PMLL diode to the optical injection of 10-Gbps PRBS NRZ data signals. ECL: ex-ternal cavity laser. PC: polarization controller. PRBS: pseudo-random binary sequence. EDFA: erbium-doped fiber amplifi er. OBPF: optical bandpass filter. VOA: variable optical attenuator. ESA: electrical spectrum analyzer. RTO: real-time oscilloscope. CLK: electrical clock signal. 

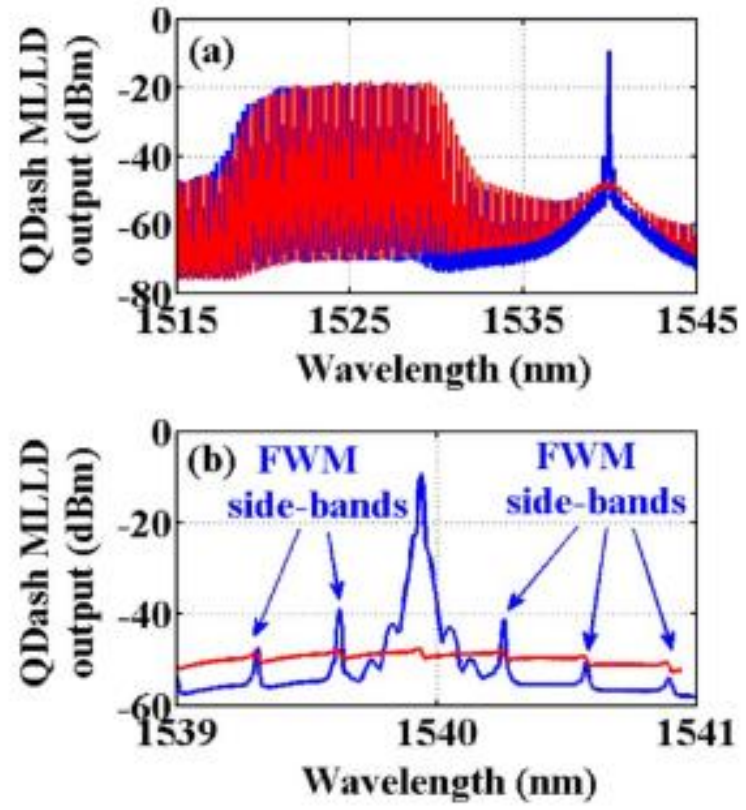

Fig. 4. Optical spectra measured at the output of the QDash PMLL diode for both synchronized (traces in blue) and de-synchronized (traces in red) conditions. (a), (b) Recorded traces for 30- and 2-nm spans, respectively. Resolution bandwidth of the utilized optical spectrum analyzer is set to $0.02 \mathrm{~nm}$. 

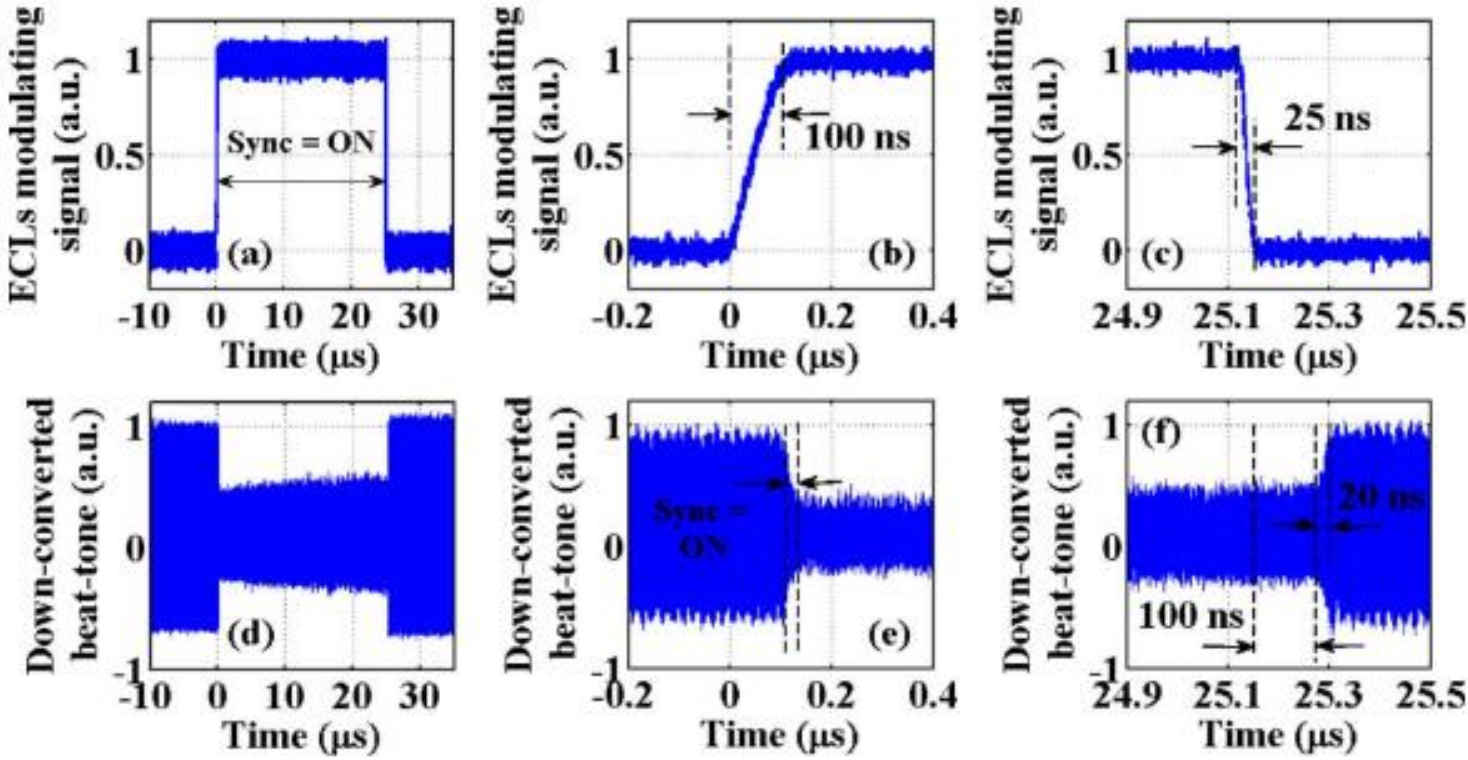

Fig. 5. (a) Normalized electrical square pulse signal utilized for modulating the intensity of the ECL. (b), (c) Zoom-in traces illustrating the rising and falling times of the modulating signal, respectively. (d) Normalized frequency down-converted beat- tones measured at the output of the RF-mixer. (e), (f) Zoom-in traces of the beat-tones illustrating the synchronization and desynchronization characteristic times, respectively. 


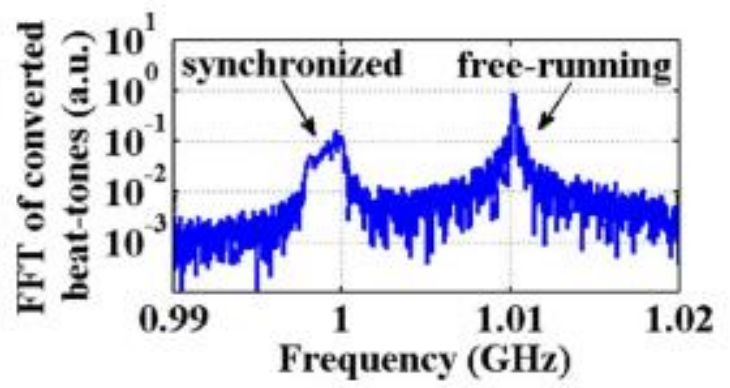

Fig. 6. FFT computed from the recorded FDC beat-tones for a time span of $50 \mathrm{~s}$. Only one spectral component is retrieved for the switching on $(0$ to $\sim 25 \mu \mathrm{s})$ and off $(\sim 25$ to $50 \mu \mathrm{s})$ times, respectively. 
